\title{
II. Ueber die Azimuthdifferenz doppeltgebrochener Strahlen. Beobachtungen am Calcit.
}

\author{
Von
}

A. Schrauf in Wien.

(Mit 2 Holzschnitten.)

Wenn von den zwei Strablen die Rede ist, welche die Doppelbrechung der einaxigen Krystalle hervorruft, dann begnügt man sich meist. mit der Angabe des Vibrationsazimuthes gegen die Hauptaxe. Der Spracbgebrauch gestattet selbst die Licenz, von zwei zu einander senkrecht polarisirten Strahlen zu sprechen, und bei prismatischer Zerlegung wird nur in den seltensten Fällen deutlich durch Worte hervorgeboben, dass die A z i mu thdifferenz, d. i. der Winkel zwischen den Vibrationsrichtungen des ordinären und extraordinären Strables $90^{\circ}$ sein kann — wie dies bekanntlich die Theorie verlangt. Beinahe fur alle Punkte der optischen Disciplin einaxiger Körper sind bereits die experimentellen Beweise geführt, nur für die Auswerthung der Azimuthdifferenz und deren Abhängigkeit von Welle und Hauptaxe fehlen dieselben.

Vor mehreren Jahren habe ich zum Zwecke der Demonstration veränderlicher Schwingungsrichtungen bei prismatischer Doppelbrechung passende Galcitprismen schleifen lassen. Die mit denselben im Laufe der Zeit erhaltenen Beobachtungen gestalten sich nun zu einer Verification des oben erwähnten Gesetzes der Schwingungsrichtuugen.

Bei diesen Untersuchungen wurden nebenher einzelne Resultate erhalten, welche $\omega, \varepsilon$, - Ausdennung - effectives Minimum der Deviation, beobachtet an Calcit, betreffen. 


\section{\$ 1. Orientirung der verwendeten Prismen aus isländischem Doppel- spath; deren Volumgewicht und mittlere lineare Ausdehnung.}

Caleitprismen sind nur dann zur Demonstration veränderlicher Vibrationsrichtungen tauglich, wenn sie drei Bedingungen genügen. Einerseits müssen sie starke Doppelbrechung zeigen, andererseits eine solche Neigung der Hauptaxe gegen die Incidenzflächen haben, dass die Azimuthe der Vibrationen bei Aenderungen des Incidenzwinkels beträchtlich variiren, schliesslich soll die krystallographische Orientirung genau möglich sein. Im Wesentlichen genugt den Bedingungen eine solche Lage der brechenden Kanten, dass diese durch die Spaltflächen gerade abgestumpft werden. Um die Beobachtungen controliren zu können, wurden aus Material einer Sendung zwei Prismen hergestellt, und eine solche Anordnung des Schliffes getroffen, dass je drei der Flächen nahe gleiche krystallographische Orientirung besitzen und in dieselben morphologischen Ebenen fallen.

Die krystallographische Bestimmung aller Schlifflächen wurde auf die Spaltflächen basirt und nach den Principien des orthohexagonalen Systems durchgeführt.

Bei künstlichen Prismen muss ferner der Schliff controlirt werden in Beziehung auf Concavilät oder Convexität der Schlifflächen. Dies ist nur möglich, wenn alle drei brechenden Flächen und die Basisfläche geschliffen, und die Neigungen letzterer gegen erstere ermittelt sind. Dieser Vorgang sollte bei allen Prismen eingehalten werden - auch bei den Glasprismen; die bisher geübte Methode des Schliffes - nur die zwei Seitenflächen an grösseren Prismen - ist deshalb ungenugend.

In den nachfolgenden Zeilen sind die Winkelverhältnisse der beiden Prismen I. und II. zusammengestellt. Die brechenden Seitenflächen sind

Fig. 1.

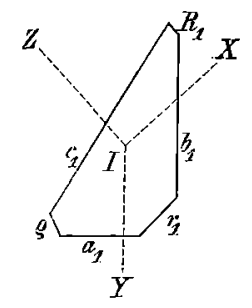

Fig. 2.

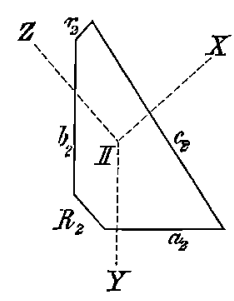

$$
\begin{aligned}
& r_{1}=(11 T) \\
& R_{1}=(1 T T)
\end{aligned}
$$
mit den Buchstaben $a_{1} b_{1} c_{1}-a_{2} b_{2} c_{2}$, die oberen Endflächen derselben direct mit den Ziffern $I, I I$, sowie die angespaltenen Rhomboëderflächen mit $r R \varrho$ bezeichnet. Die Flächen $r_{1} r_{2}$ ebenso $R_{1} R_{2}$ liegen parallel; nur fallen die Normalen von $r_{1} R_{1}$ in negative, von $r_{2} R_{2}$ in positive Quadranten. Die orthohexagonalen Indices sind daher für

$$
\begin{aligned}
r_{2} & =(T T 1) \\
\varrho_{1}=R_{2} & =(T T 1)
\end{aligned}
$$

In den obenstehenden Figuren sind die analogen Flächen auch parallel gestellt, und die Lage der Hauptaxe $Z$, sowie die der orthohexagonalen Nebenaxen $Y X$ angedeutet. 


\begin{tabular}{|c|c|c|c|}
\hline \multicolumn{4}{|c|}{ Prisma I. } \\
\hline 10 Cels.: & & $\tau$ & Beobachtet: \\
\hline 40 & $a_{1} \varrho_{1}$ & $\overline{-}$ & Q \\
\hline 20,1 & $a_{1} b_{1}$ & $=$ & 912451. \\
\hline 19,1 & $b_{1} r_{1}$ & $=$ & $42 \quad 17 \quad 52$ \\
\hline 18,7 & $b_{1} R_{1}$ & $=$ & 33715 \\
\hline 18,6 & $b_{1} I$ & $=$ & $91 \quad 4 \quad 27$ \\
\hline 20,1 & $c_{1} R_{1}$ & $=$ & 11132.9 \\
\hline 18,5 & $c_{1} \varrho_{1}$ & $=$ & $68 \quad 37$ 51 \\
\hline 20,1 & $c_{1} I$ & $=$ & $8825 \cdot 34$ \\
\hline 18,7 & $I r_{1}$ & $=$ & $93 \quad 21 \quad 15$ \\
\hline 18,8 & $I R_{1}$ & $=$ & $95 \quad 31 \quad 11$ \\
\hline 19,1 & $I \varrho_{1}$ & $=$ & $\begin{array}{lll}84 & 28 & 18\end{array}$ \\
\hline
\end{tabular}

Prisma II.

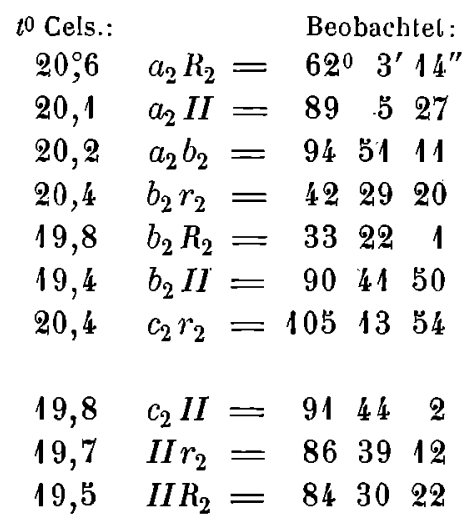

Hieraus folgt durch Rechnung:

$$
\begin{array}{r|r}
t^{0}=19,04 \quad r_{1} R_{1}=74^{\circ} 34^{\prime} 48^{\prime \prime}, 4 & t^{0}=19,99 \quad r_{2} R_{2}=74^{0} 54^{\prime} 52^{\prime \prime}, 9 \\
Z r_{1}=443626,6 & Z r_{2}=443629,5 \\
\triangleleft Z b c=792659 & \triangle Z c b=475549 \\
\sqrt{3}: 1: 0,8542374 & \sqrt{3}: 1: 0,8542614
\end{array}
$$

Aus diesen Daten ergeben sich alle für die optische Orientirung der Hauptschnitte nothwendigen Zahlen. Es folgen hier zunächst die Winkel der Schliffflächen zu den Coordinatenaxen und dann die Indices derselben. Für das Prisma I. gilt $t=19^{\circ}$ Cels., für II. hingegen $t=20^{\circ}$ Cels.

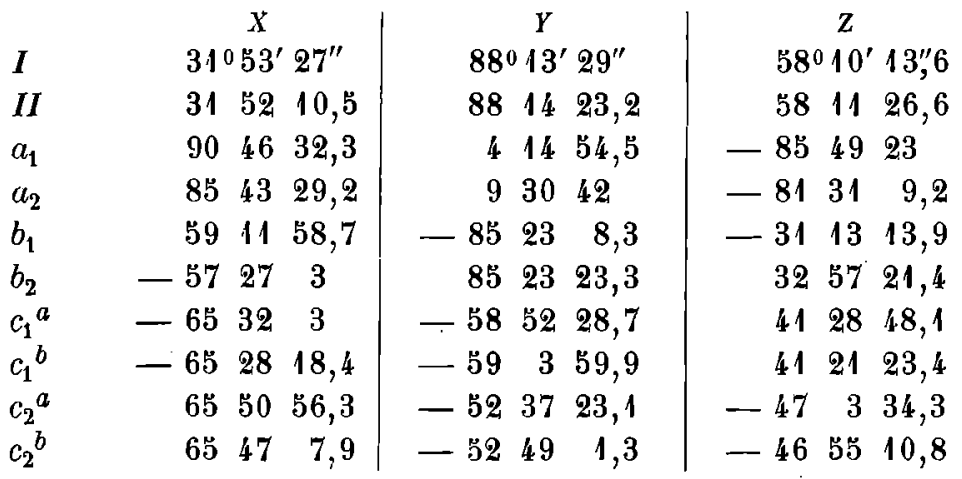

$$
\begin{aligned}
& \begin{array}{lrrlc} 
& h & & k & l \\
I & 47,470550 & : & 1: & 14,342620 \\
I I & 47,887340: & 1: & 14,658920 \\
a_{1} & -0,0235111: & 1:-0,0623914 \\
a_{2} & 0,1309201: & 1:-0,1277406 \\
b_{1} & 11,024449: & -1:-1,080865
\end{array}
\end{aligned}
$$




$$
\begin{array}{lrlrl} 
& h & k & l \\
b_{2} & -11,594035 & : & 1: & \mathbf{8}, 9180895 \\
c_{1}{ }^{a} & -1,387720 & :-1: & 1,238088 \\
c_{1}{ }^{b} & -1,398811 & :-1: & 1,247376 \\
c_{2}{ }^{a} & 1,1673675: & -1: & -0,9586529 \\
c_{2}{ }^{b} & 1,1754649 & :-1:-0,9654480
\end{array}
$$

Die grösseren Schliffflächen $c_{1} c_{2}$ sind etwas concav, wie aus den nachfolgenden Daten ersichtlich ist. Deshalb ist es nöthig, die an $a$ oder $b$ grenzenden Theile von $c$, welche die optisch wirksamen sind, als separate Flächensegmente $c_{1}{ }^{a} c_{1}{ }^{b}-c_{2}{ }^{a} c_{2}{ }^{b}$ mit bestimmten Indices zu bezeichnen. Die Controle des Schliffes erfolgt — weil die Normalen der Flächen $a, b, c$, nicht in eine Zone fallen - mit Zuhülfenabme der Messungen von $I: a$, $I: b$ u. s. w., wodurch die um die Flächennormale $I$, eventuell $I I$ gebildeten Zonenwinkel erhalten werden. Diese sind an dem

$$
\begin{aligned}
& \text { Prisma I. } \\
& \triangle c_{1} I a_{1}=124^{0} 21^{\prime} \quad 0^{\prime \prime} \quad c_{2} I I a_{2}=117^{0} 52^{\prime} 27^{\prime \prime \prime} 7 \\
& c_{1} I b_{1}=144 \quad 2428 \quad c_{2} I I b_{2}=1472846 \\
& \frac{a_{1} I b_{1}=9126 \quad 6,4}{\Sigma=360^{0}+0^{0} 11^{\prime} 34_{, 4}^{\prime \prime}} \quad \frac{a_{2} I I b_{2}=945034,4}{\Sigma=360^{0}+0^{0} 11^{\prime} 48^{\prime \prime 1}}
\end{aligned}
$$

Die Summe der Körperwinkel beträgt also bei beiden Prismen $179^{\circ} 48,5$, wodurch die Concavilät des Schliffes bewiesen ist. Ich habe die Prismen in vorliegender Gestalt trolz dieses Mangels verwendet, weil ein vorhergehendes dreimaliges Neuschleifen, Poliren und Neuberechnen der Prismen mich von der Fruchtlosigkeit fernerer Bestrebungen, absolut ebene

\begin{tabular}{|c|c|c|c|}
\hline$l^{2}$ & \multicolumn{2}{|c|}{ Beobachtet: } & $\mathrm{Ge}$ \\
\hline 0 & $a_{1} c_{1}$ & $124021^{\prime} 54^{\prime \prime}$ & $124^{\circ} 21^{\prime}$ \\
\hline & $a_{1} c$ & $124 \quad 2214$ & 242156, \\
\hline 6 & $o_{1} c$ & 14425 & $4424 \quad 44$, \\
\hline 0 & $b_{1} c$ & 1442420 & $144 \quad 24 \quad 10,2$ \\
\hline 9 & $a_{2} c$ & $11752 \quad 44$ & 1175240 \\
\hline 20 & $a_{2} c_{2}$ & 1475316 & 1175315,5 \\
\hline 4 & $b_{2} c_{2}$ & 14724 & 1472358,4 \\
\hline & $b_{2} c_{2}$ & 1472333 & 1472330 \\
\hline
\end{tabular}
Flächen in der gewünschten Lage zu erhalten, überzeugt hatte*).

Berucksichtigt man die oben angefuhrten Indices von $c^{a}, c^{b}$, dann stimmen die aus den Indices und dem Axenverhältnisșe berechneten »brechenden Winkel « fast vollkommen mit den beobachteten Werthen uberein.

*) Aus diesen Daten und der absoluten Grösse der Prismen ergiebt sich der Krümmungsradius zu $17 \mathrm{~m}$. Es ist dies eine so geringe Krümmung, dass dieselbe wabrscheinlich nicht beim Schleifen, sondern erst beim Poliren des weichen Calcits hervorgerufen ward. Zum Vergleiche hebe ich hervor, dass Voigt (diese Zeitschr. 1881, 5, 121) den Krümmungsradius eines Prisma von Zinkblende sogar mit $3,7 \mathrm{~m}$ ermittelte. 
Die Berechnung dieser Winkel für verschiedene Temperaturen basirt auf dem Axenverhältniss :

$$
x: y: z=\sqrt{3}: 1:(0,8537134+0,000027484 t),
$$

in welcher Formel $t$ die jeweilig geltende Temperatur in Celsiusgraden bedeutet.

Der hier verwendete Ausdehnungscoëfficient der Hauptaxe wurde direct abgeleitet aus den Beobachtungen Mitscherlich's*). Die Uebereinstimmung der beobachteten und gerechneten Winkelvariationen lässt denselben, wie nicht anders zu erwarten war, als vollkommen genau erscheinen.

Dass nach den Nebenaxen Contraction bei Temperaturerhöhung eintritt, folgt in Uebereinstimmung mit allen fruheren Autoren auch aus meinen Bestimmungen des Volumgewichtes der verwendeten Prismen. Ich beobachtete $\left.{ }^{* *}\right)$ :

Prisma I. Totalgewicht $5,08392 \mathrm{~g}$.

$$
t=5,21 \quad \begin{aligned}
d & =2,71247 \\
d_{4}^{5,2} & =2,712434
\end{aligned} \quad t^{\prime}=23,56 \quad \begin{aligned}
d^{\prime} & =2,71838 \\
d_{4}^{23,56} & =2,711584
\end{aligned}
$$

hieraus : linearer Ausdehnungscoëfficient pro 10. $\alpha_{I}=0,000005694$.

Prisma II. Totalgewicht 4,99095 g.

$$
t=7,92 \quad \begin{array}{rlrl}
d & =2,71407 & t^{\prime}=22^{\circ}, 81 & d^{\prime}=2,71969 \\
d_{4}^{7,92}=2,713761 & & d_{4}^{22,81}=2,713260
\end{array}
$$

hieraus: linearer Ausdehnungscoëfficient pro 10. $\alpha_{I I}=0,000004132$. Aus der Combination $\alpha_{I}+\alpha_{I I}$ der mittlere $\left.{ }^{* * *}\right)$ cubische Ausdehnungscoëfficient $3 \alpha^{10}=0,000014739$, schliesslich der Contractionscoëfficient nach den Nebenaxen zu $\alpha_{a}=-0,00000637$.

\section{\$. Ueber die Azimuthdifferenz doppeltgebrochener Strahlen.}

Den ersten directen Hinweis auf die gegenseitige Neigung von den Vibrationsrichtungen der doppeltgebrochenen Strahlen gab Biot: "Dans tout les expériments de double réfraction les deux rayons se sont toujours trouvés polarisés suivant deux directions rectangulaires. « B i ot's Versuche uber die Exlinction der doppeltgebrochenen Strahlen beim Drehen der

*) Mitscherlich, Pogg. Ann. 10, 142. $\Delta\left(r r^{\prime}\right)$ pro $100^{\circ}=8^{\prime} 34^{\prime \prime}$.

**) Zur Reduction wurden die Volumgewichte des Wassers nach Volkman (Wiedem. Ann. 14, 260) benutzt. Der Unterschied des Volumgewichtes der beiden Prismen ist durch Fissuren im Innern des Prisma I erzeugt.

***) Den mittleren cubischen Ausdehnungscoëfficienten bestimmle Mits cherlich - Dulong (Pogg. Ann. 10, 148) zu 0,00001961; Kopp (Pogg. Ann. 86, 157) zu 0,000018 ; Fizeau (C. r. 1868,66, 1012) zu 0,00001521. Die Contraction nach den Nebenaxen bestimmt F iz eau zu $\alpha^{\prime}=-0,00000562$. 
Platte und constantem Azimulh des einfallenden Lichtes führten zu folgendem Satz*): „Le rayon disparaitra . . a au lieu de faire tourner l'axe de la lame... d'une quantité egal a $-\alpha$ on pourrait le faire tourner de $-\left(\alpha+90^{\circ}\right)-\left(\alpha+180^{\circ}\right)-(\alpha+2700) . "$ Diese Biot'sche Regel enthält also die Angabe, dass die beiden doppeltgebrochenen Strahlen wirklich senkrecht zu einander polarisirt wären. $\mathrm{N}$ e u $\mathrm{mann} \mathrm{n}^{* *}$ ) hat bereits vor 50 Jahren auf die Unrichtigkeit dieser Biot'schen Angabe hingewiesen: "Die beiden Azimuthe $\alpha^{\prime} \alpha^{\prime \prime}$ stehen nicht auf einander senkrecht, wie man aus der von Biot gegebenen Regel hătte erwarten sollen. Diese Regel entfernt sich aber überhaupt für Einfallswinkel, die nicht sehr klein sind, sehr stark von der Wirklichkeit . . B Bei gegebenem Einfallswinkel $\varphi$ und gegebenem Azimuth $\omega$ der Einfallsebene kann man immer durch Wabl des Azimuthes der Polarisationsebene des einfallenden Strahles entweder den gewöhnlichen oder ungewöhnlichen Strahl verschwinden lassen.« Soll der ordentliche Strahl verschwinden, so hat man ${ }^{* * *}$ ) fur das Azimuth $\alpha^{\prime}$ der ursprünglichen Polarisationsebene

$\operatorname{tang} \alpha^{\prime}=\cos \left(\varphi-\varphi^{\prime \prime}\right) \frac{C \sin \varphi^{\prime \prime}-A \cos \omega \cos \varphi^{\prime \prime}}{A \sin \omega}+\frac{\gamma^{\prime \prime} \sin \left(\varphi^{\prime}+\varphi^{\prime \prime}\right) \sin \left(\varphi^{\prime}-\varphi^{\prime \prime}\right)}{A \sin \omega \sin \left(\varphi+\varphi^{\prime \prime}\right)}$ soll der ungewöhnliche Strahl verschwinden, so ist das Azimuth $\alpha^{\prime \prime}$

$$
\operatorname{tang} \alpha^{\prime \prime}=-\cos \left(\varphi-\varphi^{\prime}\right) \frac{A \sin \omega}{C \sin \varphi-A \cos \omega \cos \varphi^{\prime}} .
$$

Die Richtigkeit dieser zwei 1835 publicirten Gleichungen hat Neu$\mathrm{mann}$ in seiner bekannten Abhandlung über die Intensităt des ordentlichen und ausserordentlichen Strahles 1837 bewiesen, zu welchem $Z_{\text {wecke }}$ er ein combinirtes Goniometer benutzte. Seine Beobachlungen an Calcit beweisen mit einem Fehler von nur $\pm 4^{\prime}$ die Richtigkeit seiner Formeln. So erhält er beispielsweise für den Incidenzwinkel $45^{\circ}$ die Werthe von

$$
\begin{aligned}
& a^{\prime}=72^{0} 38^{\prime} \text { beobachtet } \quad 72^{0} 36^{\prime} \text { gerechnet } \\
& \alpha^{\prime \prime}=-1527,8 \quad-\quad-1526 \quad-
\end{aligned}
$$

Da Ne u ma n n seine Versuche in anderer Absicht begann, so legt er auch auf die gegenseitige Neigung der Vibrationen $\alpha^{\prime} \alpha^{\prime \prime}$ kein Gewicht und bemerkt nur nebenher $\dagger$ ): "dieses"Azimulh $\alpha^{\prime \prime}$ steht ungefähr senkrecht auf dem Azimuth $\alpha^{\prime}$ '. Obwohl also in dieser citirten Abhandlung Ne umann nicht mit ausdrucklichen Worten darauf hinweist, dass die Azimuthdifferenz der doppeltgebrochenen Strahlen von $90^{\circ}$ verschieden ist, sind doch alle daselbst publicirten Zahlen Beweise für diese Thatsache. Die Summe

*) Biot, Traité de Physique. Paris 1816, 4, 317.

**) Neumann, Abhandlungen der Berliner Akad. pro 1835. Berlin 1837, S. 61. Fassung.

***) Diese Formeln sind in der von Neumann (Pogg. Ann. 42, 11) gegebenen

†) Neumann, Pogg. Ann. 42, 1. 
der oben citirten Zahlen $79^{0} 38^{\prime}+15^{0} 2 \tau^{\prime}, 8=88^{0} 5,^{\prime} 8$ ist hierfür ein genügendes Beispiel.

Es scheint somit fast überflussig zu sein, einen vor 50 Jahren bereits bewiesenen Satz nochmals begründen zu wollen. Ich habe auch lange gezögert, ehe ich mich entschloss, die gewonnenen Zahlenreihen zu veröffentlichen. Zu dieser Entscheidung haben mich einerseits einzelne nach $\mathrm{Neu}$ m a n n's Arbeit erschienene Publicationen, andererseits auch die Erwägung gebracht, dass die von mir verwendete Art der Beobachtung und Rechnung von jener Neumann's vollkommen verschieden ist, und das erhaltene Resultat sich in Form eines einfachen Lehrsatzes zusammenfassen lässt.

Was die ersteren betrifft, so erinnere ich an Wild und Abria. Wild*) schreibt: "Diese genaue Bestätigung der Theorie (durch Neumann) beschränkt sich nur auf den Eintritt des Lichtes . . . Die Formeln für den Austritt, respective den Dụrchgang des Lichtes durch durchsichtige Körper sind damit noch nicht geprüft.، Eine totale Ignorirung der maassgebenden Arbeiten von Neumann tritt uns in der Abhandlung von A bria**) entgegen. Seine 18\%4 geschriebenen Worte: "J'ai eu l'occasion de m'assurer, qu'il y a souvent une grande différence entre les azimuths des plans de polarisation du rayon incident correspondant a l'extinction du rayon ordinaire ou extraordinaire réfracté suivant que l'incidence est normale ou oblique ", lassen erkennen, dass ihm die frutheren Arbeiten unbekannt waren, sowie ihm auch trotz seiner Versuche die markante Eigenthumlichkeit der doppeltgebrochenen Strahlen: ibre wechselnde Azimuthdifferenz, unbekannt geblieben ist. Auch wird man heutzutage kaum seine approximativen Beobachtungen, die durchschnittlich vom berechneten Azimuth des extraordinären Strahles um zwei Grade differiren (z. B. beobachtet $67^{\prime 0} 37^{\prime}$, gerechnet $64^{\circ} 36^{\prime}$ ) als das, was der Titel der Arbeit sagt, als "Vérification de la loi d'Huyghens" gelten lassen.

Der zweite mich bestimmende Grund ist die Verschiedenheit der Methode. Neumann und später Abria haben die Polarisationsverhältnisse des "einfallenden " Lichtes ermittelt, Beide haben uberdies die Lage der Hauptaxe gegen die Incidenzfläche nur approximativ bestimmt. Die vorliegenden Versuche betreffen jedoch direct die Vibrationsrichtungen der Strablen nach ihrem Austritt aus optisch vollkommen orientirten Prismen, so dass der Weg des Lichtes im doppeltbrechenden Medium und die Abhängigkeit der Vibrationsrichtung von diesem Wege sich genau angehen lässt.

Zur Beobachtung der Brechungs- und Vibrationsverhältnisse diente mir das Goniometer F u e s s Modell I, dessen Fernrohre mit exact gearbeite-

*) Wild, 1863, Pogg. Ann. 118, 195.

**) A bria, Ann. d, chim. V. Ser. 1, 311. 
ten Nicolkreisen versehen sind. Deren Diameler $0^{0} \ldots 180^{\circ}$ entspricht der Höhenlinie und deshalb auch der verticalen Prismenkante. Die fast absolute Coincidenz beider Richtungen wurde sorgfältig controlirt*); der etwa noch zu befürchtende Fehler afficirt aber die Zahlen für die Schwingungen von $\omega$ und $\varepsilon$ im entgegengeselzten Sinne und wird daher durch die Summation dieser Zahlen eliminirt. Die Azimuthdifferenz ist also von diesem Fehler unabhängig.

Da die Theilung der Nicolkreise von $0^{0} \ldots 360^{\circ}$ durchgeführt ist, so musste eine für die vorliegende Untersuchung passende Angabe der Winkel gewählt werden. Die Ablesungen in dem Quadranten $0^{\circ} \ldots 90^{\circ}$ sind mit + , jene in dem Quadranten $270^{\circ}-360^{\circ}$ mit minus bezeichnet, aber für letztere statt $360^{\circ}-\alpha$ direct $-\alpha$ angegeben worden. Dies stimmt im Wesentlichen mit dem Vorschlage von $\mathrm{Ne} \mathrm{u} \mathrm{mann}{ }^{* *}$ ) überein. In unserem Falle sind die positiven Ablesungen im Sinne des Uhrzeigers von der mit $0^{0}$ zusammenfallenden Kantenrichtung weggezählt. Die Nonien der Nicolkreise erlauben $5^{\prime}$ anzugeben, allein die Genauigkeit einer Einstellung auf das Intensilätsminimum ist immer geringer, als bei den anderen jetzt ublichen feineren stauroskopischen Methoden und der mittlere Febler einer solchen Beobachlung mindestens 10. Dieser reine Beobachtungsfehler ward jedoch durch zahlreiche Repetitionen ( $40-50 \mathrm{Mal}$, bei mehrmaliger Einstellung derselben Incidenz zu verschiedenen Zeiten) zu einem Minimum gemacht. Die so erhaltenen Mitlelwerthe sind rechnungsgemäss auf $\pm 10^{\prime}$ genau. Hierdurch ist aber der Effect unvermeidlicher Störungen nicht ausgeschlossen, unter welchen die mehrfachen Reflexe eine grosse Rolle spielen. Auch verringert sich die Genauigkeit mit Zunahme der Deviation und Verbreiterung des Strahles. Die Beobachtungen wurden durchgeführt bei Natriumlicht, bei absolut genau bestimmter Incidenz und zwar diese von $5^{0} \mathrm{zu} 5^{0}$ zunehmend, mit Benutzung jeder der beiden den brechenden Winkel einschliessenden Prismenflächen als Incidenzfläche und umfassen Polarisation und Deviation.

Die nachfolgenden Tabellen enthalten die Resultate der Beobachtung und Rechnung für die Prismen: I. mit den Flächen $b_{1} c_{1}-$ II. mit den Flächen $b_{2} c_{2}$. Angegeben ist die jedesmalige Incidenzfläche $F_{i}$ und der Incidenzwinkel $i$ auf eben diese FJäche bezogen. Dann die Deviationen $D_{\omega} D_{\mu}$ fur den ordentlichen und ausserordentlichen Strahl, sowie $r_{\omega} r_{\mu}$ die gerechneten inneren Brechungswinkel. Letztere wurden aber nicht auf die wechselnden Incidenzflächen, sondern bei jedem Prisma nur auf je eine dieser Flächen bezogen, damit sich diese Zahlen in eine fortlaufende Reihe ordnen lassen. Diese nach dem bekannten Satze $A=r+r^{\prime}$ reducirten

*) Theilweise mit Benutzung der Methode von La urent, C. r. 1878, 86, 662 .

$\left.{ }^{* *}\right)$ Ne u ma nn, Pogg. Ann. 42, 8. 
Ueber die Azimuthdifferenz doppeltgebr. Strahlen. Beobachtungen am Calcit. 13

Werthe der Brechungswinkel beziehen sich bei I. alle auf die Fläche $b$ und sind deshalb mit $r^{b}$ bezeichnet, hingegen bei II. alle auf $c$ und tragen deshalb die Bezeichnung $r^{c}$. Aus $\omega, \mu$ wurde der Hauptbrechungsexponent $\varepsilon$ gerechnet. Das Vibrationsazimuth ist mit $\alpha^{\prime}$ für $\omega$, mit $\alpha^{\prime \prime}$ für $\mu$ bezeichnet und deren Differenz in der Columne $\alpha^{\prime}-\alpha^{\prime \prime}$ angeführt. Die beobachteten Zahlen sind in Antiqua, die gerechneten in Cursiv gedruckt. Die Rechnungsoperationen werden im nächstfolgenden Paragraphen besprochen.

\section{\$ 3. Beobachtungen der Refractions- und Polarisationsverhältnisse.}

Prisma I. $b_{1} c_{1}=35^{\circ} 35^{\prime} 40^{\prime \prime}, t^{0}=18^{\circ}-21^{0}$ Cels.

a. Die Brechungsexponenten.

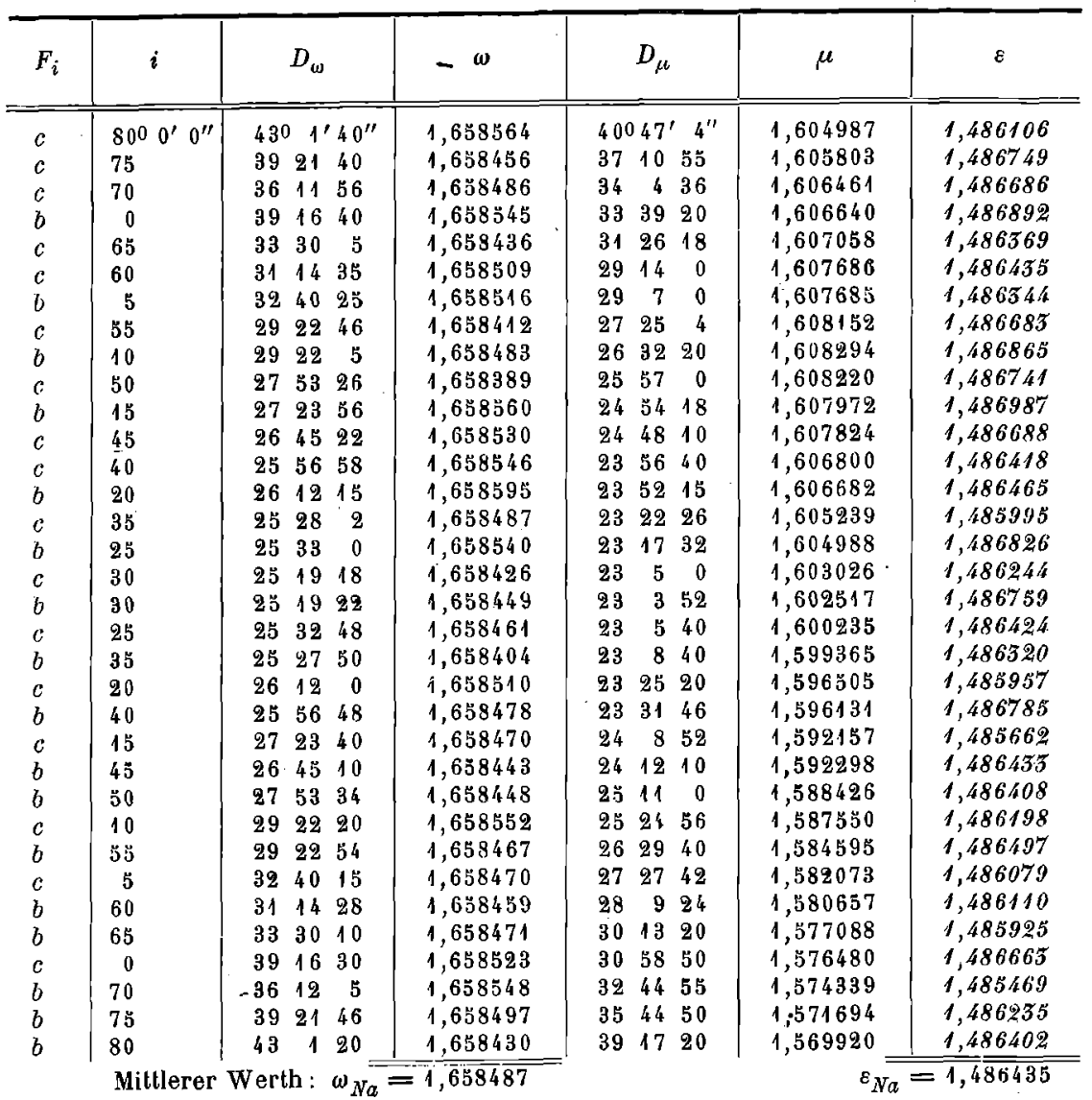


b. Die Vibrationsrichtungen:

\begin{tabular}{|c|c|c|c|c|c|c|c|c|c|c|c|c|c|c|c|c|}
\hline \multirow{3}{*}{$\underbrace{\boldsymbol{F}_{\boldsymbol{i}}}$} & \multirow{3}{*}{$\begin{array}{c}i \\
800\end{array}$} & \multicolumn{3}{|c|}{$r_{\mu}^{b}$} & \multicolumn{3}{|c|}{$\begin{array}{c}\text { Beob. } \alpha^{\prime \prime} \\
\text { Gerechn. } \alpha^{\prime \prime}\end{array}$} & \multicolumn{3}{|c|}{$r_{\omega}^{b}$} & \multicolumn{3}{|c|}{$\begin{array}{c}\text { Beob. } \alpha^{\prime} \\
\text { Gerechn. } \alpha^{\prime \prime}\end{array}$} & \multicolumn{3}{|c|}{$\begin{array}{l}\text { Peob. } \alpha^{\prime}-\alpha^{\prime \prime} \\
\text { Gerechn. } \alpha^{\prime}-a^{\prime \prime}\end{array}$} \\
\hline & & -20 & $15^{\prime}$ & $18^{\prime \prime}, 9$ & & 025 & & - $0^{0}$ & $049^{\prime}$ & $50^{\prime \prime}, 3$ & 102 & $2040^{\prime}$ & & $88^{0}$ & $01 \mathbf{5}^{\prime}$ & \\
\hline & & & & & & & $9 \quad 5^{\prime \prime}$ & & & & 101 & 53 & 24 & 87 & 44 & $1 g^{\prime \prime}$ \\
\hline \multirow[t]{2}{*}{$c$} & 75 & -1 & 23 & 4,3 & 18 & 234 & & -0 & 1 & 36,5 & 101 & 40 & & 88 & 46 & \\
\hline & & & & & & 246 & $6 \quad 40$ & & & & 100 & 35 & 39 & 87 & 48 & 59 \\
\hline \multirow[t]{2}{*}{$c$} & 70 & -0 & 12 & 17 & 1 & 43 & & 1 & 4 & 52,3 & 99 & 12 & & 88 & 27 & \\
\hline & & & & & & 52 & 256 & & & & 98 & 47 & 7 & 87 & 54 & 11 \\
\hline \multirow[t]{2}{*}{$b$} & 0 & 0 & & & -1 & $4 z$ & & 0 & 0 & & -100 & 28 & & 89 & 46 & \\
\hline & & & & & -1 & 53 & 1 & & & & -100 & 33 & 1 & 90 & 0 & \\
\hline \multirow[t]{2}{*}{$c$} & 65 & 1 & 15 & $\mathbf{5 3}$ & & 345 & & 2 & 228 & 6,1 & 96 & 40 & & 87 & 55 & \\
\hline & & & & & & & $9 \quad 3$ & & & & 96 & 29 & 34 & 88 & 0 & 21 \\
\hline \multirow[t]{2}{*}{$c$} & 60 & 3 & 0 & 2,7 & & 10 & & 4 & 6 & 59,2 & 94 & 5 & & 88 & 55 & \\
\hline & & & & & & 36 & 620 & & & & 93 & 43 & 25 & 88 & 7 & $s$ \\
\hline \multirow[t]{2}{*}{$b$} & $\mathbf{5}$ & 3 & 6 & 27,5 & - & 5 & 5 & 3 & 0 & 49,3 & -95 & 20 & & 90 & 15 & \\
\hline & & & & & & 325 & 538 & & & & -95 & 35 & 2 & 90 & 9 & 24 \\
\hline$c$ & 55 & 4 & 58 & 20,2 & & $15 \%$ & & 5 & 59 & 41 & 91 & 0 & $?$ & & $?$ & \\
\hline & & & & & & 17 & 753 & & & & 90 & 35 & 0 & 88 & 17 & 7 \\
\hline$b$ & 10 & 6 & 11 & 3,5 & & 35 & & 6 & 0 & 36,7 & -90 & 30 & & 89 & 55 & \\
\hline & & & & & & ) 16 & 610 & & & & -90 & 35 & 15 & 90 & 17 & $\ddot{z}$ \\
\hline$c$ & 50 & 7 & 8 & 54,4 & - & 95 & & 8 & 4 & 59,9 & 87 & 25 & & 88 & 10 & \\
\hline & & & & & & 125 & 2 & & & & 87 & 2 & 24 & 88 & 25 & 26 \\
\hline$b$ & 15 & 9 & 15 & 45,5 & & 515 & & 8 & 858 & 39,8 & -84 & 58 & & 90 & 13 & \\
\hline & & & & & & 456 & 6 & & & & -85 & 32 & 31 & 90 & 28 & 37 \\
\hline$c$ & 45 & 9 & 30 & 14 & - & +50 & & 10 & 21 & 20,9 & 83 & 50 & & 88 & 40 & \\
\hline & & & & & 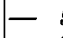 & 20 & 018 & & & & 83 & 14 & 16 & 88 & $3 / 4$ & 34 \\
\hline$c$ & 40 & 12 & 0 & 49,1 & - & 915 & & 12 & 247 & 30 & 79 & ว & & 88 & 20 & \\
\hline & & & & & & 929 & 925 & & & & 79 & 14 & 58 & 88 & 43 & 57 \\
\hline$b$ & 20 & 12 & 17 & 26,9 & & 920 & & 11 & 154 & $\quad 1,3$ & -80 & 55 & & 90 & 20 & \\
\hline & & & & & & 956 & 634 & & & & -80 & 41 & 34 & 90 & 38 & 8 \\
\hline$c$ & 35 & 14 & 39 & 32,5 & -1 & 334 & & 15 & 21 & 40,2 & 74 & 45 & & 88 & 39 & \\
\hline 1 & & & & & -13 & 344 & 429 & & & & 75 & 9 & 50 & 88 & 55 & 59 \\
\hline$b$ & 25 & 15 & 16 & 0,8 & 14 & 455 & & 14 & 45 & 45,1 & -75 & 35 & & 90 & 30 & \\
\hline & & & & & 14 & 41 & 142 & & & & -76 & 5 & 4.2 & 90 & 47 & 24 \\
\hline$c$ & 30 & 17 & 25 & 10,7 & -17 & 735 & & 18 & 2 & 50,2 & 71 & 8 & & 88 & 43 & \\
\hline & & & & & -17 & 759 & 922 & & & & 71 & 4 & 33 & 89 & 3 & 55 \\
\hline$b$ & $\mathbf{3 0}$ & 18 & 10 & 49,3 & is & $9 \mathbf{3 3}$ & & 17 & 732 & 48,7 & -71 & 2 & & 90 & 35 & \\
\hline & & & & & & 97 & $7 \quad 15$ & & & & -71 & 49 & 11 & 90 & 56 & 27 \\
\hline$c$ & 25 & 20 & 16 & 52 & -21 & 1. 35 & & 20 & 49 & 52,3 & 67 & 15 & & 88 & 50 & \\
\hline & & & & & -2 & 28 & 853 & & & & 67 & 5 & 2 & 89 & 13 & 55 \\
\hline$b$ & 35 & 21 & 0 & 57,4 & 23 & 23 & & 20 & 14 & 3,6 & -67 & 74 & & 91 & 8 & \\
\hline & & & & & 25 & 310 & $\begin{array}{ll}0 & 18\end{array}$ & & & & $-6 \%$ & 55 & 4 & 91 & 5 & 22 \\
\hline$c$ & 20 & 23 & 13 & 26,6 & -2 & 35 & & 23 & 41 & 36,4 & 63 & 16 & & 88 & 51 & \\
\hline & & & & & -26 & 68 & 2 & & & & 63 & 15 & 31 & 89 & 23 & 33 \\
\hline$b$ & 40 & 23 & 44 & s3,1 & 26 & 618 & & 22 & 48 & 13,7 & -64 & 26 & & 91 & 14 & \\
\hline & & & & & 26 & 648 & 8 41 & & & & -64 & 24 & 59 & 91 & 15 & 40 \\
\hline$c$ & 15 & 26 & 14 & 20,4 & -29 & 958 & & 26 & 636 & 58,4 & 59 & 45 & & 89 & 43 & \\
\hline & & & & & -29 & 953 & $5 \cdot 46$ & & & & 59 & 59 & 24 & 89 & 55 & 10 \\
\hline$b$ & 45 & 26 & 21 & 52,3 & .30 & 024 & & 25 & 14 & 14,2 & -61 & 0 & & 91 & 24 & \\
\hline & & & & & 30 & 22 & 245 & & & & -61 & 19 & 3 & 91 & 21 & 46 \\
\hline$b$ & 50 & 28 & 50 & 0,7 & 32 & 252 & & 27 & 730 & 36,3 & -58 & 14 & & 91 & 6 & \\
\hline & & 1 & & & 3 & 252 & 215 & & & & -58 & 37 & 0 & 91 & 29 & 13 \\
\hline$c$ & 10 & 29 & 18 & 52,7 & -39 & 40 & & 29 & 935 & 4,2 & 55 & 55 & & 89 & 35 & \\
\hline & & & & & -33 & 323 & 347 & & & & 56 & 18 & 45 & 89 & 42 & 32 \\
\hline$b$ & 55 & 31 & 7 & 39,7 & 35 & 38 & & 29 & 35 & 55,1 & -55 & 56 & & 91 & 44 & \\
\hline & & & & & $\boldsymbol{3 5}$ & 17 & 714 & & & & -56 & 17 & 51 & 91 & 35 & 5 \\
\hline$c$ & 5 & 32 & 26 & 11,2 & $\begin{array}{l}-36 \\
-36\end{array}$ & $\begin{array}{ll}6 & 38 \\
6 & 36\end{array}$ & $\begin{array}{l}8 \\
630\end{array}$ & 32 & 34 & 55,4 & $\begin{array}{l}52 \\
53\end{array}$ & $\begin{array}{l}57 \\
14\end{array}$ & 49 & $\begin{array}{l}89 \\
8.9\end{array}$ & $\begin{array}{l}33 \\
51\end{array}$ & 19 \\
\hline
\end{tabular}


Ueber die Azimuthdifferenz doppeltgebr. Strahlen. Beobachtungen am Calcit. 15

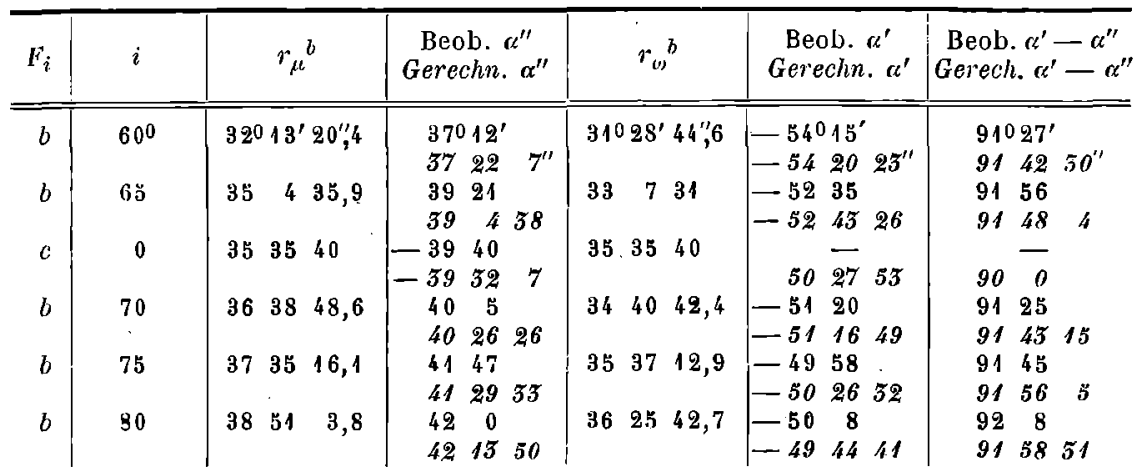

Prisma II. $b_{2} c_{2}=32{ }^{0} 36^{\prime} 27^{\prime \prime} \quad t=18^{\circ}-21^{\circ}$.

a. Die Brechungsexponenten.

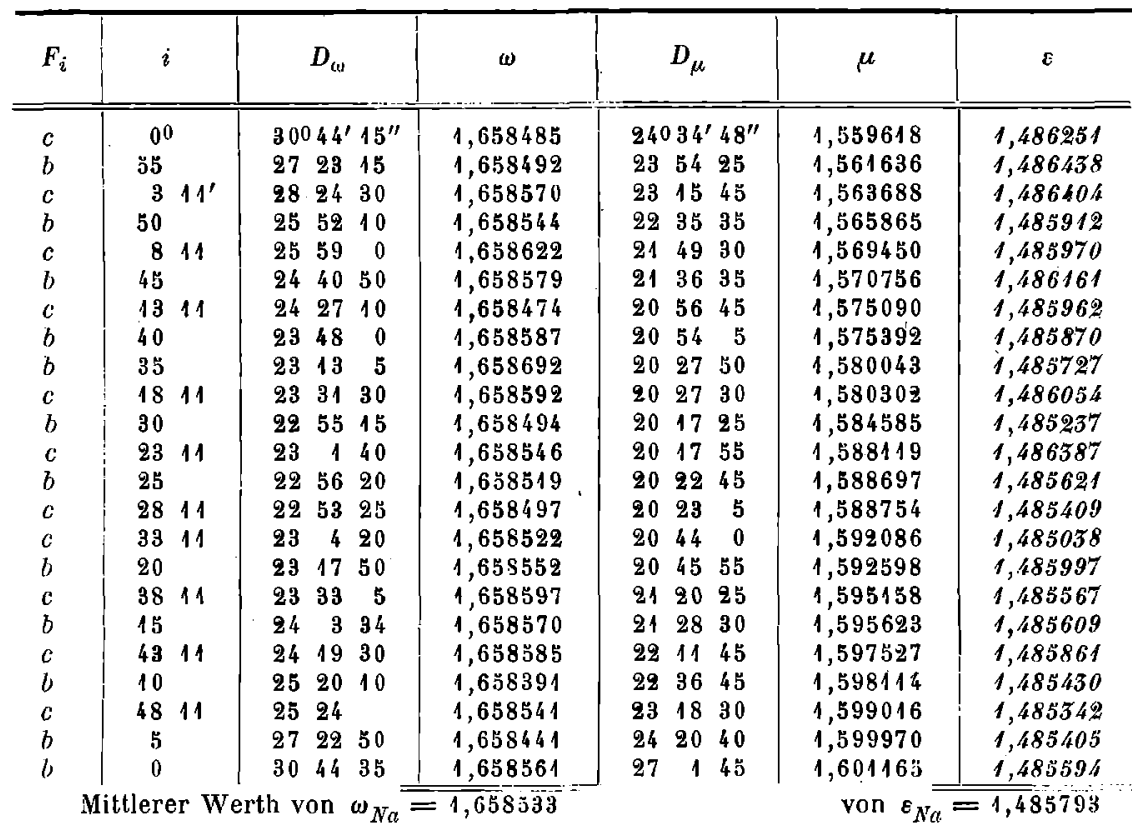

b. Die Vibrationsrichtungen.

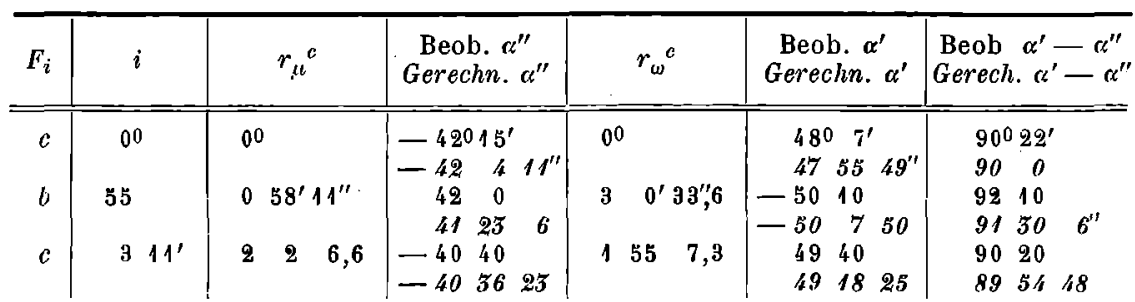




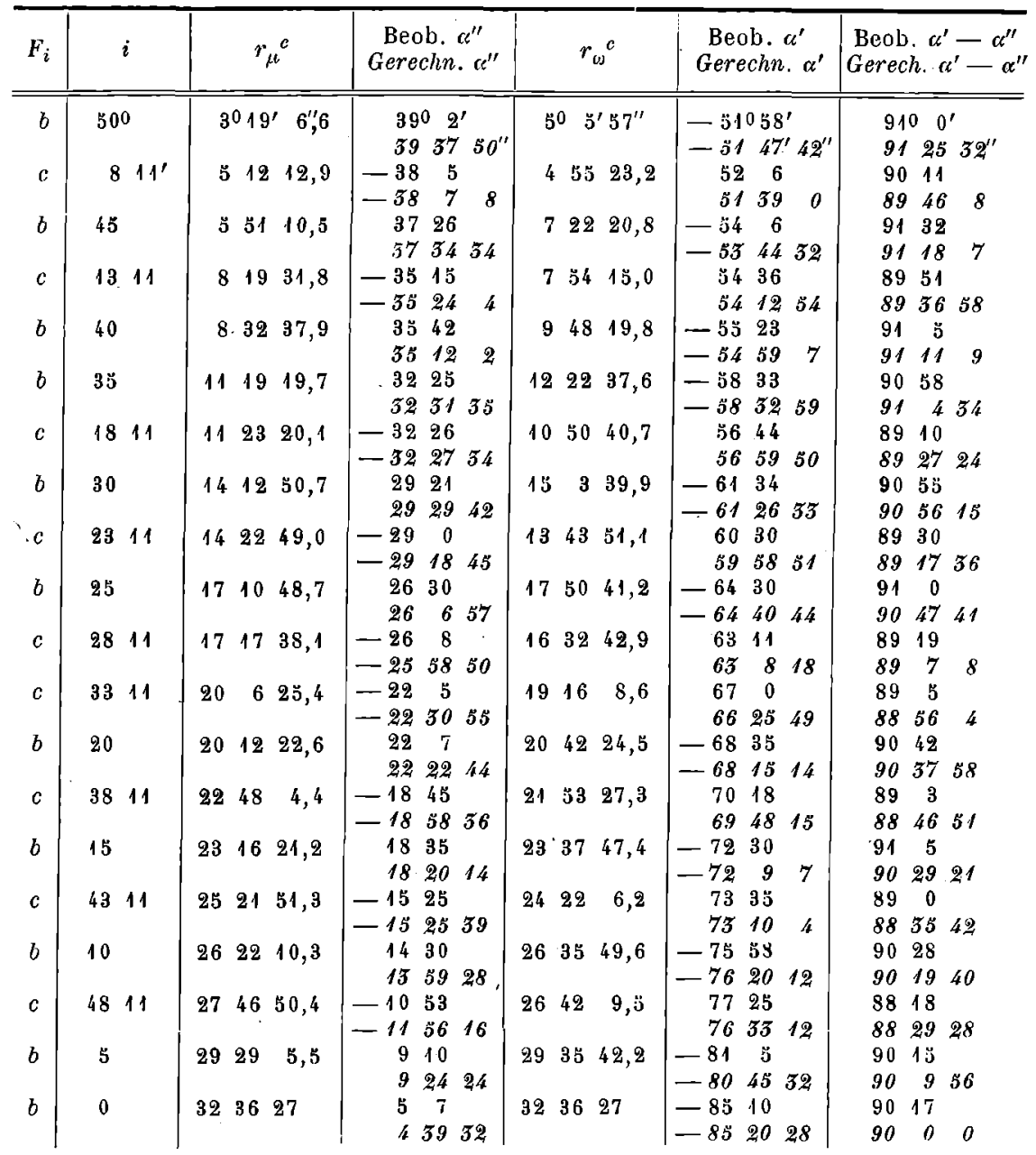

\section{\$ 4. Rechnungsformeln und Discussion der Resultate.}

a) Zur Berechnung des Hauptbrechungsexponenten $\varepsilon$ aus den beobachteten Werthen $\omega$ und $\mu$ wurde folgende Formel benutzt:

worin

$$
\varepsilon=\mu \frac{\sin \mathfrak{M}}{\sin \frac{\mathfrak{D}}{D}}
$$

$$
\mu \sin A \cos \mathfrak{M}=\omega \sin A \cos \mathfrak{D}=\cos \zeta_{i} \sin i^{\prime}-\cos \zeta_{a} \sin i \quad \text { Ia }
$$
und die Neigung der Hauptaxe zur Incidenzflàche mit $\zeta_{i}$, zur Austrittfläche mit $\zeta_{a}$ bezeichnet, der Incidenzwinkel $i$ und $A+D=i+i^{\prime}$ ist.

Da mit Natriumlicht und breiter Spaltöffnung beobachtet ward, der 
brechende Winkel überdies klein ist, ferner die Bestimmung der Brechungsexponenten nur als Nebenoperation erschien, so war hier von vornherein eine absolute Genauigkeit von $\varepsilon$ weder zu erwarten, noch anzustreben. Genauere Bestimmungen von $\varepsilon$ wurden mit anderen Flächencombinationen derselben Prismen durchgeführt und sind im ersten Anhange zusammengestellt. Die Mittelwerthe von $\omega$ und $\varepsilon$, welche aus den vorstehenden Tabellen resultiren, sind aber so nahe den von $\mathrm{Rudberg,} \mathrm{Mascart,}$ Willigen, Sarasin ... erhaltenen Zahlen, dass sie als genügende Beweise fur die Richtigkeit des ermittelten Weges der Lichtstrahlen im Medium gelten mussen. Man kann daher behaupten, die Beobachtungen sind fast absolut genau gegen die Hauptaxe orientirt. Dies ist aber jene Bedingung, von deren Erfallung das Vorausberechnen der Polarisation des Strahles abhängt.

b) Die beobachteten Vibrationsrichtungen zeigen eine gesetzmässige Abhängigkeit von der Fortpllanzungsrichtung des Lichtes im Krystall und sind für idente Richtungen - trotz wechselnder Incidenzfläche und Incidenzwinkel - gleich. Ueberblickt man den Gesammtverlauf der Reihen, dann erkennt man die vollkommene Concordanz zwischen Rechnung und Beobachtung.

Bezeichnet man mit dem Ausdrucke Transversalebene jene auf die Fortpflanzungsrichtung senkrechte Ebene $\left(T r_{i}=90^{\circ}\right)$, in welcher die Lichtschwingungen erfolgen, dann kann man die Resultate der Untersuchung durch den Satz ausdrucken:

"Die Vibrationen erfolgen für $\mu$ parallel, für $\omega$ senkrecht zu jener Linie in der Transversalebene, welche der Projection der Hauptaxe auf die jeweilige - für $\omega$ und $\mu$ wechselnde - Transversalebene entspricht.

Die Vorausberechnung der Vibrationsrichtung erfolgt deshalb nach folgender Formel, zu deren Verständniss beifolgende sphärische Projection dienen mag. In letzterer entspricht der Punkt $Z$ der Hauptaxe, $K$ der brechenden Kante, $F_{i}$ dem Einfallslothe, der Bogen $F_{i} r_{i}$ dem Werthe des inneren Brechungswinkel $r_{i}$ und deshalb ist der Winkel $90^{\circ}-Z r_{i} F_{i}$ gleich dem Azimuth von der Projection der Hauptaxe auf die Transversalebene TT, wenn dieses Azimuth von der Kante $K$ weggezählt wird. Da die Neigung der Incidenzflächen zur Haupt-

Fig. 3.

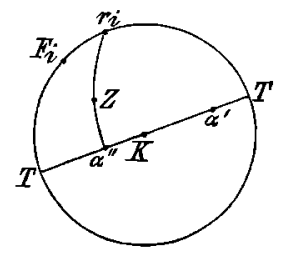
axe $\triangle F_{i} Z$ (fruher schon mit $\zeta_{i}$ bezeichnet), sowie der Winkel des Hauptschnittes $\varphi=\Varangle r_{i} F_{i} Z$, aus den krystallographischen Angaben des $\S 1$ folgen, so erhält man

*) Vergl. Schra uf, Phys. Min. 2, Formel 214. 
für $\mu$

für $\omega$

$$
\operatorname{tang} \alpha^{\prime \prime}=\frac{\operatorname{cotang} \zeta \sin r_{\mu}-\cos r_{\mu} \cos \varphi}{\sin \varphi},
$$

$$
\operatorname{cotang} \alpha^{\prime}=\frac{\operatorname{cotang} \zeta \sin r_{\omega}-\cos r_{\omega} \cos \varphi}{\sin \varphi},
$$

wodurch die Orientirung der Vibrationen gegen die brechende Kante erhalten wird. In Fig. 3 deuten die Punkte $\alpha^{\prime \prime}$ und $\alpha^{\prime}$ dieselben an, und aus deren Lage - rechts, links von $K$ - sind auch die Vorzeichen von $\alpha^{\prime}$ und $\alpha^{\prime \prime}$ zu ersehen. Diese gerechneten Werthe von $\alpha^{\prime}, \alpha^{\prime \prime}$ sind in die vorhergehenden Tabellen mit Cursivschrift unter den entsprechenden Beobachtungen eingefügt.

Schliesslich bebe ich ausdrücklich hervor, dass die fraglichen Vibrationsrichtungen auch dadurch ermittelt werden können, wenn man nach dem Vorgange von Neumann jenes Azimuth des einfallenden Strahles aufsucht, für welches die Intensität von $\omega$ oder $\mu$ gleich Null ist. Wie ich mich vielfach úberzeugt habe, erhält man in allen Fällẹn gleiche Werthe des Azimuthes und dieselbe Azimuthdifferenz, ob man den eintretenden $\left(\alpha_{i}\right)$ oder austretenden Strahl $(\alpha)$ analysirt. Nur sind die Vorzeichen vom Azimuth $\alpha_{i}$ jenein von $\alpha$ entgegengesetzt, weil die Nicolkreise am Collimator und Beobachtungsfernrohr entgegengesetzt - gegen die brechende Kante - orientirt sind.

c) A z imuthdifferenz. Die Betrachtung der obigen Formeln fur $\alpha^{\prime}, \alpha^{\prime \prime}$ lehrt unmittelbar, dass die Differenz beider $90^{\circ}$ ist, also die Vibrationen zu einander senkrecht sind, wenn $r_{\omega}=r_{\mu}$ wird, gleichgültig wie die Incidenz-Fläche oder Winkel ist. Einen solchen Fall finden wir in der Tabelle vom Prisma $\mathrm{I}$, wenn wir $\alpha^{\prime \prime}$ von $c, i=25^{0}$ mit $\alpha^{\prime}$ von $b, i=35^{0}$ vergleichen.

Ferner entnimmt man den Formeln, dass mit Zunahme von $r_{i}$ die Azimuthdifferenz $\gtreqless 90^{\circ}$ werden muss. Aus dem Differentiale der Functionen II und III :

worin

$$
\begin{aligned}
d \alpha^{\prime \prime} & =\left(1-\sin ^{2} \alpha^{\prime \prime}\right) \Psi_{\mu} d r_{\mu} \\
-d \alpha^{\prime} & =\sin ^{2} \alpha^{\prime} \Psi_{\omega} d r_{\omega},
\end{aligned}
$$

$$
\Psi_{\omega, \mu}=\left(\operatorname{cotang} \zeta \sin \varphi^{-1} \cos r+\operatorname{cotang} \varphi \sin r\right)_{r=r_{\mu} r_{\omega}}
$$

ist, wird ersichtlich, dass die Werthe von $\alpha^{\prime}, \alpha^{\prime \prime}$ im entgegengesetzten Sinne (mit dem inneren Brechungswinkel $r$ ) variiren. Dass dies thatsächlich zutrifft, zeigt ein Blick auf die Tabellen. Wenn ferner für $i=0^{0}$ die Relation $\alpha^{\prime}-\alpha^{\prime \prime}=90^{\circ}$ gilt, so wird bei Zunahme von $i$ die Azimuthdifferenz nur wenig, soweit der Einfluss der obigen Factoren, die nahezu gleich sind, reicht, sich von $90^{\circ}$ unterscheiden können. 
Bildet man andererseits - mit Berücksichtigung der effectiven Vorzeichen - die Differenz $\left(\alpha^{\prime}-\alpha^{\prime \prime}\right)$ aus den Gleichungen II, III, und zwar dadurch, dass man in Annäherung statt der Functionen die Winkel, sowie für cotang $\zeta \cos \varphi$ die Constanten $C^{\prime}$ und $C^{\prime \prime}$ einführt - so erhält man folgende Relation :

$$
\Delta=90^{\circ}-\left(\alpha^{\prime}-\alpha^{\prime \prime}\right)=\left(r_{\omega}-r_{\mu}\right) C^{\prime}+C^{\prime \prime}(\ldots
$$

diese besagt: in erster Annäherung entspricht der Transgredient, d.i. der Unterschied der Azimuthdifferenz von $90^{\circ}$, dem Unterschiede der inneren Brechungswinkel für ordinäre und extraordinäre Strahlen.

Als Beispiele hierfur können die ganzen Tabellen der Vibrationsbestimmungen gelten. Nur einige Zahlen will ich hier wiederholen:

$$
\begin{array}{llll}
\text { Prisma II. } & c, i=28^{\circ} & \Delta=52^{\prime} 55^{\prime \prime} & r_{\omega}-r_{\mu}=44^{\prime} 56^{\prime \prime} \\
& c, i=48 & \Delta=1^{0} 30^{\prime} 32^{\prime \prime} & r_{\omega}-r_{\mu}=1^{\prime} 4^{\prime} 41^{\prime \prime}
\end{array}
$$

Dadurch hat man ein einfaches Mittel zur Verfügung, um den Minimalwerth des Transgredienten: also der Differenz der Extinctionsrichtungen zweier doppeltgebrochener Strahlen von $90^{\circ}$ voraussagen zú können, weil die Factoren $C^{\prime}, C^{\prime \prime}$ sich nur wenig von der Einheit unterscheiden.

Wenn bei stauroskopischen Untersuchungen nicht genau senkrechte Incidenz stattfindet, wird auch daselbst der Werth des Transgredienten zu berücksichtigen sein.

Die letztgewonnene Gleichung hat ibr Analogon in dem theoretischen Lehrsatze von von Lang*), welchen Hollefreund ${ }^{* *}$ ) neuerdings reassumirt. Lang beweist: "der zur schnelleren Welle gehörige Strahl steht senkrecht auf der Schwingungsrichtung der langsameren und umgekehrt."

\section{Anhang $\mathbf{I}$.}

Die Hauptbrechungsexponenten $\omega, \varepsilon$, von Calcit. Die Flächencombinationen $a_{1} c_{1}=55^{\circ} 37^{\prime} 46^{\prime \prime}$ und $a_{2} c_{2}=62^{\circ} 6^{\prime} 44^{\prime \prime} \mathrm{der}$ nach $\S 1$ krystallographisch orientirten Prismen I, II erwiesen sich geeignet, um mit einiger Genauigkeit die Dispersionsverhältnisse von $c, \varepsilon$ zu revidiren. Die Beobachtungen wurden mit variabler Incidenz durchgeführt, und die nachfolgenden Zahlen stellen das Mittel von 19maliger Durchmusterung des ganzen Spectrum dar. $\varepsilon$ wurde aus $\mu$ mittelst Formel I berechnet. Für die Fraunhofer'schen Linien wurden die Wellenlängen

$\left.{ }^{*}\right)$ v on Lang, Sitzungsber. der Wiener Akad. 1861, 43, Formel 65. ,

$\left.{ }^{* *}\right)$ Hollefreund, Nova Act. Leopold. Halle 1883, 46, 20. 
nach Ditscheiner, fưr Lithium und Thallium nach Thalén benutzt; uberdies in der Dispersionsformel $\lambda_{D}{ }^{\prime \prime}=0,5891$ gesetzt.

Das totale Mittel aller Beobachtungen ist :

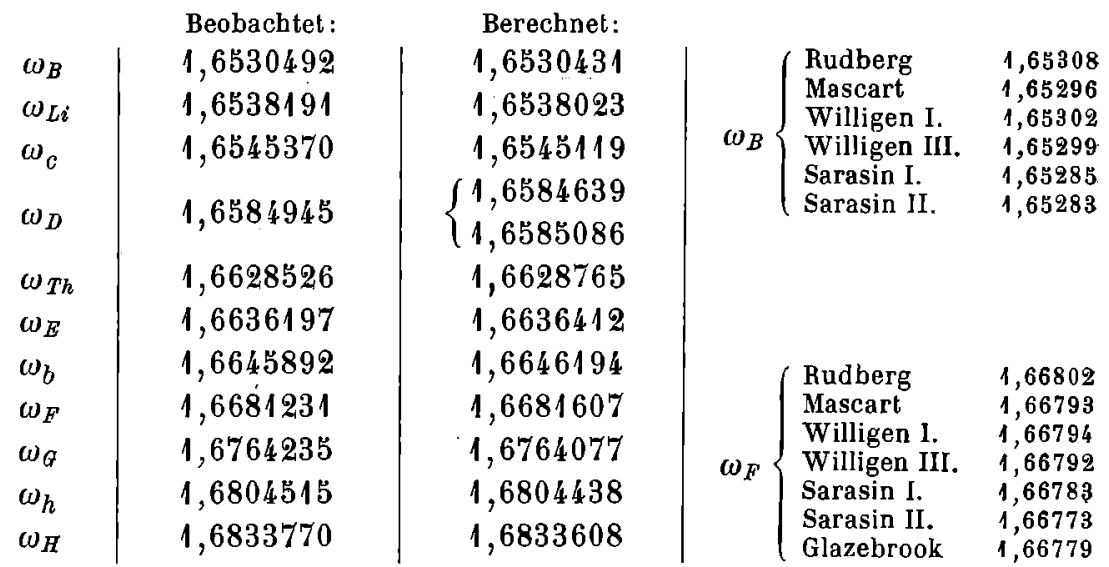

Die Dispersionsformel, welche aus diesen beobachteten Werthen von $\omega$ folgt, lautet:

$$
\begin{gathered}
\omega=0,00024954 \lambda^{2}+1,637698+0,00720696 \lambda^{2}-0,0000054196 \lambda^{-4} \\
+0,00000005802 \lambda^{-8} .
\end{gathered}
$$

Fur den Hauptbrechungsexponenten $\varepsilon$ erhielt ich durch Rechnung aus $\omega$ und den jeweiligen $\mu$ folgende Zahlen $\left.{ }^{*}\right)$ :

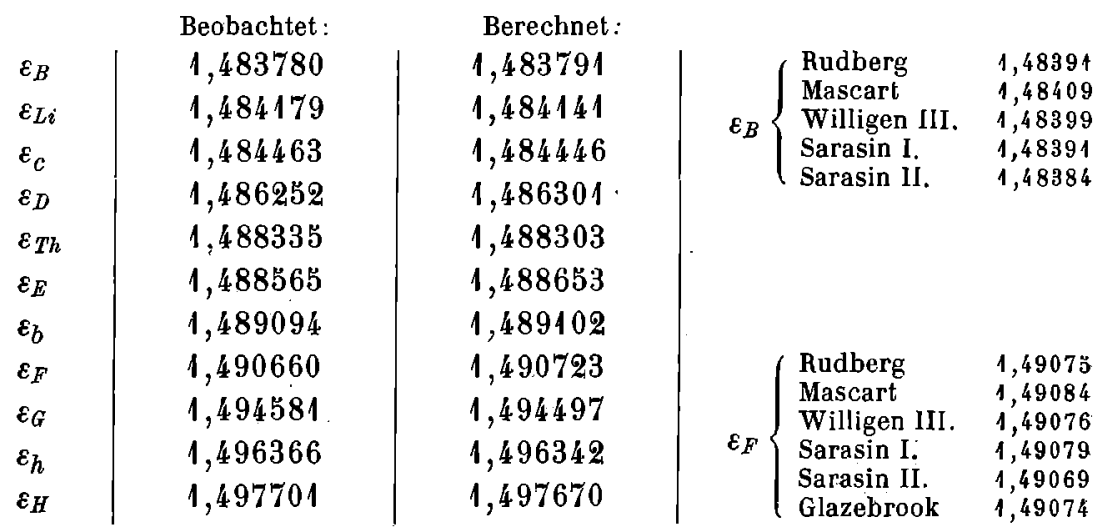

*) Ma s cart, Ann. sc. d. l'écol. norm. Paris 1864, 1, 237. Willigen, Arch. d. Mus. Teyler II-III, 1870, 61. G lazebrook, Phil. Transact. 1880, 171, 421. S a rasin, C. r. 1882, 95, 680. Diese Zeitschr. 9, 605. 
Die gerechneten Zahlen für $\varepsilon$ basiren auf der Dispersionsformel:

$$
\varepsilon=1,476851+0,0272305 \lambda^{-2} \text {. }
$$

Die vorstehenden Beobachtungen gelten für die mittlere Temperatur $t=20^{\circ}, 5$ Celsius, und beziehen sich auf $\mathrm{CaCO}_{3}$ mit dem mittleren Volumgewicht $d \frac{20,5}{20,5}=2,71712$ oder $d \frac{20,5}{4}=2,71253$.

\section{Anhang II.}

Effectives Minimum der Deviation des ausserordentl i chen Strahles. Das theoretische Minimum der Deviation setzt voraus, dass $r=\cdot r^{\prime}=\frac{A}{2}$ sei. Mit diesem theoretischen Minimum fällt das beobachtete effective Minimum der Deviation bei dem ausserordenllichen Strahle nur dann zusammen, wenn die Halbirungslinie des brechenden Winkels in den optischen Hauptschnitt fällt. Den Beweis hierfür hat von Lang*) gegeben.

Die Differenz der Ablesungen fur die Deviationen bei effectivem und theoretischem Minimum ist eine Function der Wellenfläche des betreffenden Mediums. Für Körper mit schwacher Doppelbrechung ist dieser Winkelunterschied kaum bemerkbar, bei Substanzen mit starker Doppelbrechung, wie es Spath ist, beträgt er circa 1 Minute. Da es für manche Untersuchungen von Nutzen sein kann, genau uber die Grösse des Fehlers orientirt zu sein, welchen man begeht, wenn man $\mu$ des effectiven Minimum mit $\mu^{\prime}$ des theoretischen Minimum gleichsetzt, deshalb hạbe ich an meinen Calcitprismen einige diesbezügliche Beobachtungsreihen durchgeführt.

Prisma I. $b_{1} c_{1}=35^{\circ} 35^{\prime} 40^{\prime \prime}$.

Theoretisches Minimum: Incidenzfläche $b_{1}$

$$
\begin{aligned}
& i=29017^{\prime} 0^{\prime \prime} \quad i=29017^{\prime} 25^{\prime \prime} \\
& D=23 \quad 4 \quad 4 \quad D=23 \quad 4 \quad 8 \\
& \mu_{N a}^{\prime}=1,602675 \quad \mu_{N a}^{\prime}=1,602702 \\
& \text { Effectives Minimum: } i=31^{0} 33^{\prime} 13^{\prime \prime} \quad i=27^{\circ} 13^{\prime} 8^{\prime \prime} \\
& D=23 \quad 3 \quad 10 \quad D=23 \quad 3 \quad 9 \\
& r_{b}=19 \quad 419,5 \quad r_{e}=16 \quad 3538,8 \\
& \mu_{N a}=1,601483 \quad \mu_{N a}=1,601566
\end{aligned}
$$

Zur Controle des effectiven Minimums kann man die Gleichung

$$
\sin i \sin r^{\prime}=\sin i^{\prime} \sin r
$$

benutzen, in welcher $i, r$ und $i^{\prime}, r^{\prime}$ auf die beiden möglichen Incidenz-

*) v o n Lang, Sitzungsber. der Akad. Wien 1858, 33, 160. 
22 A. Schrauf. Ueber die Azimuthdiff. doppeltgebr. Strahlen. Beob. am Calcit.

flächen zu beziehen sind. Wie man aus den Zahlenwerthen für $\mu$ entnimmt, beträgt der Fehler, den man begeht, wenn man theoretisches und effectives Minimum bei Calcit verwechselt, eine Einheit in der dritten Decimalstelle. Bei schwächer doppeltbrechenden Körpern wird daher die dritte Decimalstelle für beide Minimumstellungen noch ident sein. Uebrigens ist es selbstverständlich, dass das effective Minimum der Deviation nicht zugleich jenem wirklichen Minimum des ausserordentlichen Brechungsexponenten entspricht, welches dasselbe Prisma bei grösserem oder kleinerem Incidenzwinkel - je nach der Lage des Hauptschnittes zu liefern vermag.

\section{Inhalt:}

$\S 1$. Orientirung der verwendeten Prismen aus isländischem Doppelspath, deren Volumgewicht und mittlere lineare Ausdehnung.

82. Ueber die Azimuthdifferenz doppeltgebrochener Strahlen.

$\S 3$. Beobachtungen der Refraclions- und Polarisationsverhältnisse.

$\S$ 4. Rechnungsformeln und Discussion der Resultate.

Anhang I. Die Hauptbrechungsexponenten $\omega, \varepsilon$ des Calcit.

Anhang II. Effectives Minimum der Deviation des ausserordentlichen Strahles. 